\title{
Timing of Initiation of Calcineurin Inhibitors in Pediatric Haploidentical Transplantation with Post- Transplantation Cyclophosphamide: Effects on Survival, Relapse, and Cytokine Release Syndrome
}

\section{Vedat Uygun ( $\nabla$ veddat@hotmail.com )}

İstinye University, Faculty of Medicine, MedicalPark Antalya Hospital https://orcid.org/0000-0003-

3257-7798

\section{Gulsun Karasu}

İstinye University School of Medicine, MedicalPark Göztepe Hospital

Koray Yalçın

MedicalPark Göztepe Hospital

\section{Seda Öztürkmen}

MedicalPark Antalya Hospital

Hayriye Daloglu

MedicalPark Antalya Hospital

\section{Safiye Suna Çelen}

MedicalPark Göztepe Hospital

\section{Volkan Hazar}

Medipol University Faculty of Medicine https://orcid.org/0000-0002-1407-2334

Akif Yesilipek

MedicalPark Antalya Hospital

Article

Keywords: Cyclophosphamide, Calcineurin Inhibitors, Haploidentical

Posted Date: April 26th, 2021

DOl: https://doi.org/10.21203/rs.3.rs-428093/v1

License: (9) (1) This work is licensed under a Creative Commons Attribution 4.0 International License.

Read Full License 
Version of Record: A version of this preprint was published at Acta Haematologica on November 30th, 2021. See the published version at https://doi.org/10.1159/000521211. 


\section{Abstract}

The use of unmanipulated haploidentical stem cell transplantations (haplo-HSCT) with post-transplant cyclophosphamide (PTCY) in children has emerged as an acceptable alternative to the patients without a matched donor. However, the timing of calcineurin inhibitors $(\mathrm{CNI})$ used in combination with PTCY is increasingly becoming a topic of controversy. We evaluated 49 children with acute leukemia who underwent unmanipulated haplo-HSCT with PTCY according to the initiation day of CNIs (pre- or post$\mathrm{CY}$ ). There were no significant differences in the overall survival analysis between the two groups. The cumulative incidence of relapse at 2 years was $21.2 \%$ in the pre-CY group and $38.9 \%$ in the post-CY group $(p=0.33)$. Cytokine release syndrome (CRS) was observed more frequently in the post-CY group. Our study shows that the argument for starting $\mathrm{CNI}$ administration after $\mathrm{CY}$ is tenuous, and the rationale for not starting $\mathrm{CNI}$ before $\mathrm{CY}$ needs to be reconsidered.

\section{Introduction}

Recent developments in practices have made haploidentical hematopoietic stem cell transplantations (haplo-HSCTs) more frequent, although they were generally limited to those recipients who had an available matched donor. In unmanipulated settings, post-transplantation cyclophosphamide (PTCY) treatment is a widely used prophylactic method for graft-versus-host disease (GVHD) because of its low cost, high availability, and ease of application [1]. In this method, after administration of unmanipulated stem cells, proliferation of alloreactive T-cells is allowed for 3 days without any restrictive drugs. On days 3 and 4 or 5 , it was assumed that destruction of alloreactive cells occurs by cyclophosphamide (CY), and was followed by continued immunosuppression with a calcineurin inhibitor (CNI) and mycophenolate (MMF) $[2,3]$. Whether CNIs should be used after cyclophosphamide administration for not inhibiting the development of the alloreactive cell population becomes controversial because recent studies have shown that cyclophosphamide induces alloreactive $\mathrm{T}$ cell functional impairment rather than destroying them [4].

Although many previous studies have supported the use of immunosuppression after PTCY, a few studies, including ours, have shown that it can be started before PTCY, with no loss of effectiveness [2, 5, 6]. To the best of our knowledge, only one study has reported a systematic comparison of the effects of early and late introduction of immunosuppression in PTCY; earlier application (pre-CY) was associated with lower risks of relapse and chronic GVHD (cGVHD), with no differences in other transplantation outcomes [2]. However, in this retrospective multicenter study, pediatric patients were not included. In our center, we used immunosuppression pre-CY, due to the development of CGVHD in initial post-CY patients. However, as more studies showed positive results with a post-CY approach, we changed our policy to post-CY application as well [5]. In this single-center study, we compared the outcomes of pediatric patients who underwent unmanipulated haplo-HSCT with PTCY with pre- or post-CY immunosuppression.

\section{Materials And Methods}


All pediatric patients (under 20 years of age) who consecutively underwent haplo-HSCT for acute leukemia with the administration of PTCY for GVHD prophylaxis at the Medical Park Antalya Hospital (Antalya, Turkey) between March 2013 and May 2020 were retrospectively reviewed. Haplo-HSCT was performed for high-risk patients; patients were categorized as high-risk according to the treatment protocol or with a history of former rejected transplantation. Pre-transplant remission evaluation was performed and the count of remission was recorded to define the disease status before transplantation. Written informed consent was obtained from the parents of all patients in accordance with the Declaration of Helsinki. Ethical approval for this study was granted by the Ethics Board of the Medical Park Antalya Hospital.

All except 4 patients and donors were genotyped using a high-resolution molecular standard technique at their HLA-A, -B, -C, -DRB1, and -DQB1 loci. The remaining 4 patients and donors were genotyped using a low-resolution molecular technique at their HLA-A, -B, and -DRB1 loci. Although a haplo-compatible sibling with an appropriate weight is preferred as a donor, this is rarely encountered in practice. If the degree of allele match between the patient and both parents was the same, the mother was preferred as the donor. In cases of consanguinity, available relatives were also screened. Donor-specific anti-HLA antibodies that may contribute to graft failure were analyzed and, in case of positivity, other available donors were chosen.

\section{Conditioning regimens and GVHD prophylaxis}

All conditioning regimens were myeloablative. Non-TBI conditioning regimens almost always included $150 \mathrm{mg} / \mathrm{m}^{2}$ fludarabine with busulfan, according to a weight-adjusted dose (parenteral, without therapeutic drug monitoring). Occasionally, both TBI and non-TBI modalities were used in combination with various agents such as thiotepa and melphalan.

Our initial strategy for choosing the stem cell source was to obtain them from granulocyte colony stimulating factor (G-CSF)-primed, unmanipulated bone marrow (BM). With this strategy, we could harvest peripheral blood stem cells (PBSC) on the subsequent day and obtain a CD34 + cell count greater than $6 \times 10^{6} / \mathrm{kg}$. With experience, our current revised strategy utilizes peripheral blood stem cells in patients with malignancy.

For GVHD prophylaxis, all patients received high-dose $\mathrm{CY}(50 \mathrm{mg} / \mathrm{kg})$ for two days, administered on the 3rd day at the earliest and on the 5th day at the latest. Mesna (100-150\% of the CY dose) was administered from day one of CY to day seven post-HSCT. CNIs, generally with MMF were also administered for GVHD prophylaxis. In our initial patients subjected to unmanipulated haplo-HSCT with PTCY, CNI and MMF administration started on the day of transplantation or one day after transplantation, and the doses were adjusted to maintain a trough level of $100-200 \mathrm{ng} / \mathrm{mL}$ cyclosporine $A$ and $5-10$ $\mathrm{ng} / \mathrm{mL}$ tacrolimus [5]. MMF was administered orally thrice daily at $600 \mathrm{mg} / \mathrm{m}^{2}$ from day 0 . We later changed our schedule of immunosuppression to begin one day after the last dose of CY. If no GVHD was 
observed, the MMF dose was tapered off from day +30 over 2-3 months. CNI treatment was administered through the 6th month, then tapered off over three months.

G-CSF treatment $(5 \mu \mathrm{g} / \mathrm{kg} /$ day) was started on day +5 and continued until neutrophil engraftment. Neutrophil engraftment was considered to have started on the first of 3 consecutive days with an absolute neutrophil count (ANC) $\geq 0.5 \times 10^{9} / \mathrm{L}$, and platelet recovery was considered to have started on the first of seven consecutive days in which the platelet count $\geq 20 \times 10^{9} / \mathrm{L}$ without transfusion support. The diagnosis and grading of acute GVHD (aGVHD) and cGVHD were based on the modified Glucksberg criteria and $\mathrm{NIH}$ criteria, respectively $[7,8]$. Infection prophylaxis and microbiological screening were performed as described previously [5]. Neutropenic fever and viremias were treated according to the relevant guidelines, as described previously $[5,9]$.

\section{Cytokine release syndrome}

Cytokine release syndrome (CRS) was graded according to published criteria [10]. CRS-like symptoms on the day of transplantation and the first 5 days after transplantation were taken into consideration. Patients who displayed such symptoms before transplantation and continued to do so after transplantation and patients who were initially diagnosed with CRS and later revised with an infection diagnosis were not considered in the final analysis as having had haplo-HSCT-related CRS. Empiric antibiotic therapy was initiated after appropriate cultures were obtained from all patients who presented with CRS symptoms. Dexamethasone was administered at $10 \mathrm{mg} / \mathrm{m}^{2} /$ day for $1-2$ days for patients considered as having had haplo-HSCT related CRS. Regardless of the fever response, dexamethasone treatment was stopped within 2 days at the latest.

\section{Poor Graft}

During the post-transplant follow-up, eltrombopag was initiated in patients who continued to require platelet transfusion at least once every 7 days even after 60 days of transplantation or had secondary platelet failure after achieving primary platelet recovery. Eltrombopag treatment was also started in patients who required G-CSF support at least once a week after 30 days of transplantation. Systemic evaluations were routinely performed to exclude relapse of the underlying disease, viral infection, active GVHD, and other definitive causes.

Event-free survival (EFS) was defined as the probability of being alive without evidence of relapse/progression, or death from any cause. Overall survival (OS) was defined as the time from the date of transplantation to death from any cause.

All statistical analyses were performed using SPSS for Windows (version 16.0.0; SPSS Inc., Chicago, IL). Descriptive statistics of qualitative variables were expressed as frequencies and percentages. Proportions of patients with certain baseline characteristics were compared using the $\chi^{2}$ test or Fischer's exact test. The OS and EFS probabilities on the initial day of GVHD prophylaxis were calculated using the KaplanMeier method, and comparisons between probabilities in different groups were performed using the log- 
rank test. Variables with log-rank test $p$ values $<0.200$ were included in multivariate analyses (stepwise Cox proportional hazard regression analysis). Statistical significance was set at $p<0.05$.

\section{Results}

Data from 49 patients who received unmanipulated donor stem cells for haplo-HSCT and CY for GVHD prophylaxis were collected and outcomes were compared to extract the effect of the timing of CNI. The characteristics of the patients and their transplants are shown in Tables 1 and 2 . There was no significant difference between the two groups in terms of age, general condition, remission status, gender, number of transplants, and patient-donor relationship. Bone marrow was used more frequently in the pre-CY group as a stem cell source. This was because we used pre-CY application in the initial years of our haplo-HSCT experience, and bone marrow was used frequently in these transplants due to concerns about GVHD (Table 2). 
Table 1

Patient Characteristics

\begin{tabular}{|c|c|c|c|}
\hline \multirow[t]{2}{*}{ Characteristic } & \multicolumn{2}{|l|}{ Initial day of CNI } & \multirow[t]{2}{*}{$\mathbf{p}$} \\
\hline & Pre-Cy $(n=29)$ & Post-Cy $(n=20)$ & \\
\hline Age at Tx (median, years) & $10.0(1.8-20.0)$ & $9.4(4.4-15.8)$ & 0.58 \\
\hline Lansky score at Tx (median) & $70(40-100)$ & $80(60-80)$ & 0.22 \\
\hline Sex & & & 0.27 \\
\hline Male & 22 & 18 & \\
\hline Female & 7 & 2 & \\
\hline Disease & & & 0.58 \\
\hline ALL & 21 & 13 & \\
\hline AML & 8 & 7 & \\
\hline Malignant disease status & & & 0.92 \\
\hline CR1 & 4 & 2 & \\
\hline$\geq \mathrm{CR} 2$ & 18 & 13 & \\
\hline Refractory & 7 & 5 & \\
\hline Number of Tx & & & 0.52 \\
\hline 1 & 20 & 12 & \\
\hline 2 & 9 & 8 & \\
\hline Donor relation & & & 0.11 \\
\hline Mother & 19 & 7 & \\
\hline Father & 8 & 9 & \\
\hline Other & 2 & 4 & \\
\hline
\end{tabular}


Table 2

Transplant Characteristics

Initial day of CNI

Pre-Cy $(n=29) \quad$ Post-Cy $(n=20)$

Conditioning regimen

0.16

Busulfan based

$25(86 \%)$

$13(65 \%)$

TBI based

$4(14 \%)$

$7(35 \%)$

Additional GVHD prophylaxis

$\mathrm{CNI}$

$9(31 \%)$

$5(25 \%)$

$\mathrm{CNI}+\mathrm{MMF}$

$20(69 \%)$

$15(75 \%)$

Defibrotide prophylaxis

No

$22(76 \%)$

15 (75\%)

Yes

$7(24 \%)$

$5(25 \%)$

Stem cell source

BM + PBSC (median, range)

$22(76 \%)$

$10(50 \%)$

TNC in BM $(10-8 / \mathrm{kg})$

$5.8(1.9-13.8)$

$5.3(3.2-10.1)$

0.98

MNC in PBSC $(10-8 / \mathrm{kg})$

$6.8(4.7-14.3)$

$6.0(4.6-11.0)$

0.77

CD34 $(10-6 / \mathrm{kg})$

$8.4(3.7-21.0)$

$13.2(7.6-19.0)$

0.13

PBSC

$7(24 \%)$

$10(50 \%)$

MNC $(10-8 / \mathrm{kg})$

$8.0(7.1-12.5)$

$9.0(7.2-12.7)$

0.62

CD34 $(10-6 / \mathrm{kg})$

$6.6(4.0-9.1)$

$9.4(6.2-17.4)$

0.26

Engrafted patients (\%)

Neutrophil

$29(100 \%)$

19(95\%)

0.40

Platelet

$25(86 \%)$

$18(90 \%)$

0.69

Engraftment days (median, range)

Neutrophil

$15(10-30)$

$15(12-22)$

0.80

Platelet

$13(9-66)$

$22(9-79)$

0.36

TBI: total body irradiation; GVHD: graft versus host disease; CNI: calcineurin inhibitor; MMF: mycophenolate mofetil; BM: bone marrow; PBSC: peripheral blood stem cell; TNC: total nucleated cell; MNC: mononucleated cell; CRS: Cytokine release syndrome; VOD: veno-occlusive disease 


\section{Pre-Cy $(n=29) \quad$ Post-Cy $(n=20)$}

CRS (Grade 1)

No

Yes

Dexamethasone

No

Yes

Acute GVHD

Grade $0-1$

Grade 2

Grade 3-4

Chronic GVHD

No

Mild

Moderate

Severe

Complications

CMV viremia

Hemorrhagic cystitis

VOD

Bleeding

Adenovirus viremia

Other

Eltrombopag

Relapse
17 (59\%)

$10(31 \%)$

$4(14 \%)$

$3(10 \%)$

$2(7 \%)$

$5(17 \%)$

$6(20 \%)$

$5(17 \%)$
$6(30 \%)$

$4(20 \%)$

$2(10 \%)$

1 (5\%)

0 (0\%)

$3(15 \%)$

$4(20 \%)$

7 (35\%)
0.13 $<0.01$

0.04

1 (5\%)

$13(65 \%)$

$4(20 \%)$

3 (15\%)

0.59

15 (70\%)

$3(18 \%)$

$2(6 \%)$

$0(6 \%)$

(

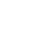




\section{Pre-Cy $(n=29) \quad$ Post-Cy $(n=20)$}

\begin{tabular}{|c|c|c|c|}
\hline Death & $14(48 \%)$ & $7(35 \%)$ & 0.36 \\
\hline \multicolumn{4}{|l|}{ Causes of Death } \\
\hline Infection & $4(14 \%)$ & $1(5 \%)$ & \\
\hline Relapse/progression & $2(7 \%)$ & $4(20 \%)$ & \\
\hline aGVHD & $2(7 \%)$ & $1(5 \%)$ & \\
\hline Hemorrhage & $2(7 \%)$ & $0(0 \%)$ & \\
\hline Other/Unknown & $4(14 \%)$ & $1(5 \%)$ & \\
\hline
\end{tabular}

None of the patients experienced CRS over grade 1, and the only manifestation of CRS was fever. CRS was observed significantly more frequently in the post-CY group $(p=0.04)$. To determine whether the high frequency of CRS was related to the stem cell source, we compared CRS rate in patients with different stem cell sources (PBSC vs BM + PBSC) regardless of the groups and observed no significant association $(p=0.38)$. Furthermore, we found that the rate of CRS had no association with nucleated cell count or CD34 + cell count ( $p=0.63$ and $p=0.13$, respectively). CRS occurred more frequently in patients subjected to TBI in the conditioning regimen ( $n=11 / 11$ vs. $29 / 38)(p=0.07)$. CRS was not associated with the emergence of aGVHD or cGVHD in both the pre-and post-CY groups.

Consistent with the high frequency of CRS in the post-CY group, dexamethasone usage was also significantly higher in the post-CY group than in the pre-CY group (Table 2). Patients administered dexamethasone in the post-CY group tended to relapse more than those who were not administered dexamethasone $(p=0.06)$. Dexamethasone usage did not increase the frequency of aGVHD or cGVHD; in fact, dexamethasone treatment was associated to a lower frequency of aGHVD $(\mathrm{OR}=0.16,95 \% \mathrm{Cl} 0.04-$ $0.64 ; p<0.01)$ and cGVHD (OR $=0.19,95 \% \mathrm{Cl} 0.04-0.99 ; p=0.04)$.

Myeloid reconstitution was achieved on day +30 for $29(100 \%)$ and $19(95 \%)$ patients in the pre-and post$\mathrm{CY}$ groups, respectively. In the post-CY group, all patients showed full donor chimerism on day +30 , except one, who died before engraftment. In the pre-CY group, one patient relapsed immediately after engraftment and the remaining patients $(96 \%)$ displayed full-donor chimerism on day +30 . No significant difference was detected in the severity of acute or chronic GVHD in either group. 
The most frequent complication in both groups was viremia of CMV (Table 2). When adenovirus- and BK virus-associated hemorrhagic cystitis together with $\mathrm{CMV}$ were considered, viremia was found to occur significantly more frequently in the pre-CY group $(\mathrm{OR}=3.2,95 \% \mathrm{Cl} 1.0-10.6 ; \mathrm{p}=0.05)$.

The frequency of relapse in the pre-CY group $(n=5 / 29 ; 17 \%)$ was lower than that in the post-CY group ( $n$ $=7 / 20 ; 35 \%)$, although this was not statistically significant $(p=0.15)$.

The median follow-up time of the surviving 28 patients was 24 months (range 9-90 months) and 19 months (range 9-46 months) in the pre-CY and post-CY groups, respectively. The OS at 2 years was 55.2 $\%(36.8-73.6 \%, 95 \% \mathrm{Cl})$ and $56.8 \%(29.2-84.4 \%, 95 \% \mathrm{Cl})$, while event-free survival (EFS) at 2 years was $51.7 \%(33.1-70.3 \%, 95 \% \mathrm{Cl})$ and $41.1 \%(14.1-68.1 \%, 95 \% \mathrm{Cl})$, in the pre-CY and post-CY groups, respectively (Figs. 1 and 2). There were no significant differences in the OS and EFS analysis between the two groups. Multivariate analyses for survival also revealed no prognostic risk factors between the two groups. Although it appeared that post-CY setting has a positive effect on OS in higher-risk groups (CR3 or refractory), this advantage was lost on EFS because of increased relapse frequency in that group.

Since almost all patients in the post-CY group experienced CRS (Table 2), we looked at the effect of CRS on survival analysis only in the pre-CY group. The OS and EFS at 2 years in patients with and without CRS in that group were $42.9 \%(21.3-64.5 \%, 95 \% \mathrm{Cl})$ vs $87.5 \%(64.1-100 \%, 95 \% \mathrm{Cl})(\mathrm{p}=0.04)$ and $38.1 \%$ $(16.9-59.3 \%, 95 \% \mathrm{Cl})$ vs $87.5 \%(64.1-100 \%, 95 \% \mathrm{Cl})(\mathrm{p}=0.04)$, respectively (Table 3$)$. 
Table 3

Results of Univariate Analysis for 2-year Outcomes

\begin{tabular}{|c|c|c|c|c|c|c|c|}
\hline & $\mathbf{n}$ & Pre-Cy $(95 \%$ Cl) & p & $\mathrm{n}$ & Post-Cy (95 \% Cl) & $\mathbf{p}$ \\
\hline \multicolumn{2}{|l|}{ os } & 29 & $55.2 \%(36.8-73.6)$ & & 20 & $56.8 \%(29.2-84.4)$ & 0.39 \\
\hline \multicolumn{2}{|l|}{ EFS } & 29 & $51.7 \%(33.1-70.3)$ & & 20 & $41.1 \%(14.1-68.1)$ & 0.97 \\
\hline \multicolumn{2}{|c|}{$\mathrm{CIR}^{*}$} & 29 & $21.2 \%(9.7-46.5)$ & & 20 & $38.9 \%(22.6-66.8)$ & 0.33 \\
\hline \multicolumn{2}{|c|}{ OS (CR1 and CR2) } & 17 & $76.5 \%(55.9-97.1)$ & & 8 & $87.5 \%(64.1-100)$ & 0.53 \\
\hline \multicolumn{2}{|c|}{ EFS (CR1 and CR2) } & 17 & $70.6 \%(48.4-92.8)$ & & 8 & $75.0 \%(44.4-100)$ & 0.88 \\
\hline \multicolumn{2}{|c|}{ OS (CR3 and refractory) } & 12 & $25.0 \%(0-50.0)$ & & 12 & $41.7 \%(8.5-74.9)$ & 0.07 \\
\hline \multicolumn{2}{|c|}{ EFS (CR3 and refractory) } & 12 & $25.0 \%(0-50.0)$ & & 12 & $25.0 \%(0.0-53.8)$ & 0.30 \\
\hline \multirow[t]{2}{*}{ OS } & CRS $(-)$ & 8 & $87.5 \%(64.1-100)$ & \multirow[t]{2}{*}{0.04} & - & - & \\
\hline & CRS (+) & 21 & $42.9 \%(21.3-64.5)$ & & - & - & \\
\hline \multirow[t]{2}{*}{ EFS } & CRS (-) & 8 & $87.5 \%(64.1-100)$ & \multirow[t]{2}{*}{0.04} & - & - & \\
\hline & CRS (+) & 21 & $38.1 \%(16.9-59.3)$ & & - & - & \\
\hline \multirow[t]{2}{*}{ OS } & Dexamethasone (-) & - & - & & 5 & $100 \%(100-100)$ & \multirow[t]{2}{*}{0.08} \\
\hline & Dexamethasone (+) & - & - & & 15 & $42.9 \%(10.7-75.1)$ & \\
\hline \multirow[t]{2}{*}{ EFS } & Dexamethasone (-) & - & - & & 5 & $100 \%(100-100)$ & \multirow[t]{2}{*}{0.03} \\
\hline & Dexamethasone $(+)$ & - & - & & 15 & $23.3 \%(0.0-49.9)$ & \\
\hline
\end{tabular}

Due to the high frequency of dexamethasone use in the post-CY group due to the high frequency of CRS occurrence, we looked at its effect on survival analysis. The OS and EFS at 2 years in dexamethasone users of post-CY group were $42.9 \%(10.7-75.1 \%, 95 \% \mathrm{Cl})$ vs $100 \%(100-100 \%, 95 \% \mathrm{Cl})(\mathrm{p}=0.08)$ and $23.3 \%(0.0-49.9 \%, 95 \% \mathrm{Cl})$ vs $100 \%(100-100 \%, 95 \% \mathrm{Cl})(\mathrm{p}=0.03)$, respectively (Table 3$)$.

\section{Discussion}

Although an increasing number of studies are reporting that unmanipulated haplo-HSCTs with PTCY are effective, the effects of conditioning regimen [11], stem cell source [12] and donor characteristics [5, 13] on the outcome remain unclear. An important point of contention is regarding the timing of $\mathrm{CNI}$ administration (pre- or post-CY) in terms of efficacy and complications $[2,5,6]$. Since factors such as conditioning regimen, stem cell source, donor gender, stem cell count, and duration of immunosuppression affect haplo-HSCTs, the haplo-HSCTs vary between patients, and more importantly, 
between centers, and thus, it is difficult to draw general conclusions. Our single-center study of 49 patients with leukemia will therefore be a useful standard to compare the results of large multi-center studies, such as the one by Ruggeri et al. [2], which included 509 patients with leukemia from 34 centers. Our results show that pre-CY $\mathrm{CNI}$ administration could potentially benefit cases of relapse in acute leukemias and a noninferiority to post-CY application in survival outcomes, similar to Ruggeri et al. In this study, we found that pre-CY application provides an advantage for patients with CRS.

Although post-CY setting had a positive effect on OS in the higher risk groups (CR3 or refractory), this effect is lost in EFS due to increased relapse frequency. The slightly negative effect on OS in the pre-CY group is possibly associated with increased infections. As the increased frequency of viremia in haploHSCTs is a known problem $[5,14]$, the increase in the pre-Cy group was higher than that in the post-CY group. The fact that early $\mathrm{CNI}$ initiation in the pre-CY group prevents $\mathrm{T}$ cell proliferation earlier and consequently for a longer time, possibly increases the non-relapse mortality.

CRS development is an expected complication in unmanipulated haplo-HSCTs $[15,16]$. Although it mostly occurs in cases where the stem cell source is peripheral stem cells, it can also occur in transplants where the stem cell source is bone marrow. As CRS diminishes shortly after $\mathrm{CY}$ administration, proliferating lymphocytes may be responsible for this phenomenon, although this has not been proven yet. In our study, this argument is also supported by the fact that the frequency of CRS in the pre-CY group was much lower and less problematic, possibly because of the direct effect of CNIs in preventing rapid proliferation of alloreactive T-cells. In our study, almost all of the patients in the post-CY group developed CRS where immunosuppressives did not start early, and they required dexamethasone because of the refractory fever, but almost no patient required dexamethasone in the pre-CY group due to the fact that it only caused a slight non-refractory fever, suggesting that the CRS development was not related solely to haploidentical feature, but due to the alloreactive cells that emerged by infused stem cells which were not prevented by an immediate efficient immunosuppressive such as cyclophosphamide or methotrexate. The fact that CRS can develop not only in haploidentical HSCTs but also in HLA-identical HSCTs with post-CY application also supports this [16]. In addition, as the rate of CRS was not related to the number of cells used, it may not only be associated with alloreactive cells, but also with organ damage developed after the conditioning regimen, as all patients with TBI developed CRS in our study.

Since almost all patients in the post-CY group developed CRS, we looked at the effect of CRS development on survival analysis in the pre-CY group and found that the development of CRS significantly reduced OS and EFS in the 2-year survival analysis, similar to recent studies $[15,17]$. Hence, it is important to prevent CRS with drugs such as dexamethasone. Routine administration of corticosteroids before PTCY is generally avoided because preventing the proliferation of alloreactive Tcells that make them susceptible to $\mathrm{CY}$ could theoretically decrease the efficacy of CY in terms of GVHD. Nevertheless, severe and refractory courses of fever, despite antibiotics, in patients with deep neutropenia necessitates the use of short-term steroids. We used dexamethasone in about $33 \%$ of the patients, mostly in the post-CY group (Table 2), and almost always had a positive response within a day. The use of dexamethasone before CY did not increase the frequency of GVHD in our study but it did increase the 
tendency to relapse in the post-CY group; thus, it significantly decreased the probability of EFS in this group. Dexamethasone has an apoptotic effect on lymphocytes, while CNIs decrease the activity of lymphocytes without triggering apoptosis in them $[18,19]$. Alloreactive cells in the pre-CY group may have eliminated the sparing effect of $\mathrm{CY}$ because of previously initiated CNIs; thus, it increased the effect of graft-versus-leukemia (GVL) and decreased the possibility of relapse (although it was not significant). However, this effect did not occur when dexamethasone was used before $\mathrm{CY}$ in the post-CY group suggesting that dexamethasone was effective in eliminating alloreactive cells completely, resulting in a decreased GVL effect. We recruited a small number of patients in our study; therefore, a larger prospective randomized study is needed to show whether dexamethasone has a negative effect on the development of GVHD or increases relapse when administered before PTCY.

Eltrombopag is a thrombopoietin receptor agonist primarily used in immune thrombocytopenic purpura. It has also been used in acquired aplastic anemia in recent years, because of its effects on trilineage hematopoiesis. Due to the similarities between the poor graft problem that develops after transplantation and acquired aplastic anemia, eltrombopag is increasingly being used in this context as well [20]. The main causes of poor graft function in an haplo-HSCT setting are conditioning, GVHD, viral infections and donor specific antibodies [21, 22]. In vivo T cell depletion using PTCY overcomes T cell- and NK cellmediated graft rejection and we observed that, in our study, pre-CY initiation of CNIs did not have a negative effect on this mechanism. Further, no effect of the timing of the initiation of CNIs in the development of poor graft function in haplo-HSCTs with PTCY was observed suggesting that poor graft development might be more related to antibody-mediated rejection rather than cell-mediated rejection.

Our study has a few limitations. First, our study population comprised a heterogeneous patient population with significant variation in disease properties, conditioning regimens, types of donors, sources of stem cells, and conditions of the recipient. Second, the lack of specific diagnostic methods for the diagnosis of CRS has resulted in a vague definition of CRS. However, it is obvious that fever is more common in post-CY patients in the days after stem cell infusion. Third, haplo-HSCTs seem to be affected by multifactorial effects, and the trend for increased relapses in patients treated with dexamethasone may not actually be directly related to dexamethasone use.

Although the dataset studied had some limitations, our study shows that the argument for starting CNI administration after $\mathrm{CY}$ is tenuous, and the rationale for not starting $\mathrm{CNI}$ before $\mathrm{CY}$ needs to be reconsidered. The timing of initiation of CNIs had no effect on survival analyses and GVHD frequency in our study; it supports the finding [4] that cyclophosphamide induces alloreactive T cell functional impairment rather than destroying them. In an unmanipulated haplo-HSCT setting, the CNI administration in a PTCY may be individualized to start before or after CY. It may be more logical to start CNIs before CY for patients with a high probability of relapse. Although dexamethasone may be useful for CRS patients, it should be avoided in high-risk patients with malignancies because of the possibility of increasing relapse.

\section{Declarations}


Conflict of interest: There are no conflicts of interest to declare

Contribution Statement of Authors: Vedat Uygun conceived and designed the analysis, collected the data, contributed data, performed the analysis, and wrote the paper; Gülsün Karasu conceived and designed the analysis, collected the data, contributed data; Koray Yalçın conceived and collected the data, and contributed data; Seda Öztürkmen collected the data, and contributed data; Hayriye Daloglu collected the data, and contributed data; Safiye Suna Çelen conceived and collected the data, and contributed data; Volkan Hazar conceived and designed the analysis, and contributed data; Akif Yeşilipek conceived and designed the analysis, and contributed data

\section{References}

1. D'Souza A, Fretham C, Lee SJ, et al. Current Use of and Trends in Hematopoietic Cell Transplantation in the United States. Biol Blood Marrow Transplant. 2020 Aug;26(8):e177-e182.

2. Ruggeri A, Labopin M, Battipaglia G, et al. Timing of Post-Transplantation Cyclophosphamide Administration in Haploidentical Transplantation: A Comparative Study on Behalf of the Acute Leukemia Working Party of the European Society for Blood and Marrow Transplantation. Biol Blood Marrow Transplant. 2020 Oct;26(10):1915-1922.

3. Luznik L, O'Donnell PV, Symons HJ, et al. HLA-haploidentical bone marrow transplantation for hematologic malignancies using nonmyeloablative conditioning and high-dose, posttransplantation cyclophosphamide. Biol Blood Marrow Transplant. 2008 Jun;14(6):641-50.

4. Wachsmuth LP, Patterson MT, Eckhaus MA, et al. Post-transplantation cyclophosphamide prevents graft-versus-host disease by inducing alloreactive T cell dysfunction and suppression. J Clin Invest. 2019 Mar 26;129(6):2357-2373.

5. Uygun V, Karasu G, Daloglu H, et al. Haploidentical hematopoietic stem cell transplantation with posttransplant high-dose cyclophosphamide in high-risk children: A single-center study. Pediatr Transplant. 2019 Nov;23(7):e13546.

6. Chiusolo P, Bug G, Olivieri A, et al. A Modified Post-Transplant Cyclophosphamide Regimen, for Unmanipulated Haploidentical Marrow Transplantation, in Acute Myeloid Leukemia: A Multicenter Study. Biol Blood Marrow Transplant. 2018 Jun;24(6):1243-1249.

7. Przepiorka D, Weisdorf D, Martin P, et al. 1994 Consensus Conference on Acute GVHD Grading [Consensus Development Conference Review]. Bone Marrow Transplant. 1995 Jun;15(6):825-8.

8. Filipovich $A H$, Weisdorf D, Pavletic $S$, et al. National Institutes of Health consensus development project on criteria for clinical trials in chronic graft-versus-host disease: I. Diagnosis and staging working group report. Biol Blood Marrow Transplant. 2005 Dec;11(12):945-56.

9. Uygun $V$, Karasu $G$, Daloglu $H$, et al. Ruxolitinib salvage therapy is effective for steroid-refractory graft-versus-host disease in children: A single-center experience. Pediatr Blood Cancer. 2020 Apr;67(4):e28190. 
10. Lee DW, Gardner R, Porter DL, et al. Current concepts in the diagnosis and management of cytokine release syndrome. Blood. 2014 Jul 10;124(2):188-95.

11. Huselton E, Slade M, Trinkaus KM, et al. Propensity Score Analysis of Conditioning Intensity in Peripheral Blood Haploidentical Hematopoietic Cell Transplantation. Biol Blood Marrow Transplant. 2018 Oct;24(10):2047-2055.

12. Ruggeri $A$, Labopin $M, B a c i g a l u p o ~ A$, et al. Bone marrow versus mobilized peripheral blood stem cells in haploidentical transplants using posttransplantation cyclophosphamide. Cancer. $2018 \mathrm{Apr}$ 1;124(7):1428-1437.

13. Stern M, Ruggeri L, Mancusi A, et al. Survival after T cell-depleted haploidentical stem cell transplantation is improved using the mother as donor. Blood. 2008 Oct 1;112(7):2990-5.

14. Slade M, Goldsmith S, Romee R, et al. Epidemiology of infections following haploidentical peripheral blood hematopoietic cell transplantation. Transpl Infect Dis. 2017 Feb;19(1).

15. Imus PH, Blackford AL, Bettinotti M, et al. Severe Cytokine Release Syndrome after Haploidentical Peripheral Blood Stem Cell Transplantation. Biol Blood Marrow Transplant. 2019 Dec;25(12):24312437.

16. Solan L, Landete E, Bailen R, et al. Cytokine release syndrome after allogeneic stem cell transplantation with posttransplant cyclophosphamide. Hematol Oncol. 2020 Oct;38(4):597-603.

17. Abboud R, Keller J, Slade M, et al. Severe Cytokine-Release Syndrome after T Cell-Replete Peripheral Blood Haploidentical Donor Transplantation Is Associated with Poor Survival and Anti-IL-6 Therapy Is Safe and Well Tolerated. Biol Blood Marrow Transplant. 2016 Oct;22(10):1851-1860.

18. Iorgulescu JB, Gokhale PC, Speranza MC, et al. Concurrent Dexamethasone Limits the Clinical Benefit of Immune Checkpoint Blockade in Glioblastoma. Clin Cancer Res. 2020 Nov 25.

19. Schreiber SL, Crabtree GR. The mechanism of action of cyclosporin A and FK506. Immunol Today. 1992 Apr;13(4):136-42.

20. Halahleh K, Gale RP, Da'na W, et al. Therapy of posttransplant poor graft function with eltrombopag. Bone Marrow Transplant. 2021 2021/01/01;56(1):4-6.

21. Ciurea SO, de Lima M, Cano $P$, et al. High risk of graft failure in patients with anti-HLA antibodies undergoing haploidentical stem-cell transplantation. Transplantation. 2009 Oct 27;88(8):1019-24.

22. Kong Y. Poor graft function after allogeneic hematopoietic stem cell transplantation-an old complication with new insights(). Semin Hematol. 2019 Jul;56(3):215-220.

\section{Tables}

TABLE 1 Patient Characteristics 


\begin{tabular}{|c|c|c|c|}
\hline Characteristic & Initial d & $\mathrm{y}$ of $\mathrm{CNI}$ & $\mathrm{p}$ \\
\hline & Pre-Cy (n=29) & Post-Cy $(n=20)$ & \\
\hline Age at Tx (median, years) & $10.0(1.8-20.0)$ & $9.4(4.4-15.8)$ & 0.58 \\
\hline Lansky score at Tx (median) & $70(40-100)$ & $80(60-80)$ & 0.22 \\
\hline Sex & & & 0.27 \\
\hline Male & 22 & 18 & \\
\hline Female & 7 & 2 & \\
\hline Disease & & & 0.58 \\
\hline ALL & 21 & 13 & \\
\hline AML & 8 & 7 & \\
\hline Malignant disease status & & & 0.92 \\
\hline CR1 & 4 & 2 & \\
\hline$\geq \mathrm{CR} 2$ & 18 & 13 & \\
\hline Refractory & 7 & 5 & \\
\hline Number of Tx & & & 0.52 \\
\hline 1 & 20 & 12 & \\
\hline 2 & 9 & 8 & \\
\hline Donor relation & & & 0.11 \\
\hline Mother & 19 & 7 & \\
\hline Father & 8 & 9 & \\
\hline Other & 2 & 4 & \\
\hline
\end{tabular}

Tx: transplantation; ALL: acute lymphoblastic leukemia; AML: acute myeloid leukemia; CR: complete remission

TABLE 2 Transplant Characteristics 


\begin{tabular}{|c|c|c|c|}
\hline & \multirow{2}{*}{\multicolumn{2}{|c|}{ Initial day of CNI }} & \multirow{3}{*}{$\mathrm{p}$} \\
\hline & & & \\
\hline & Pre-Cy $(n=29)$ & Post-Cy $(n=20)$ & \\
\hline \multicolumn{3}{|l|}{ Conditioning regimen } & \multirow[t]{3}{*}{0.16} \\
\hline Busulfan based & $25(86 \%)$ & $13(65 \%)$ & \\
\hline TBI based & $4(14 \%)$ & $7(35 \%)$ & \\
\hline \multicolumn{3}{|l|}{ Additional GVHD prophylaxis } & \multirow[t]{3}{*}{0.65} \\
\hline CNI & $9(31 \%)$ & $5(25 \%)$ & \\
\hline $\mathrm{CNI}+\mathrm{MMF}$ & $20(69 \%)$ & $15(75 \%)$ & \\
\hline \multicolumn{3}{|l|}{ Defibrotide prophylaxis } & \multirow[t]{3}{*}{0.95} \\
\hline No & $22(76 \%)$ & $15(75 \%)$ & \\
\hline Yes & $7(24 \%)$ & $5(25 \%)$ & \\
\hline \multicolumn{3}{|l|}{ Stem cell source } & \multirow[t]{2}{*}{0.08} \\
\hline BM+PBSC (median, range) & $22(76 \%)$ & $10(50 \%)$ & \\
\hline TNC in BM $(10-8 / \mathrm{kg})$ & $5.8(1.9-13.8)$ & $5.3(3.2-10.1)$ & 0.98 \\
\hline MNC in PBSC $(10-8 / \mathrm{kg})$ & $6.8(4.7-14.3)$ & $6.0(4.6-11.0)$ & 0.77 \\
\hline CD34 (10-6/kg) & $8.4(3.7-21.0)$ & $13.2(7.6-19.0)$ & 0.13 \\
\hline PBSC & $7(24 \%)$ & $10(50 \%)$ & \\
\hline MNC $(10-8 / \mathrm{kg})$ & $8.0(7.1-12.5)$ & $9.0(7.2-12.7)$ & 0.62 \\
\hline CD34 (10-6/kg) & $6.6(4.0-9.1)$ & $9.4(6.2-17.4)$ & 0.26 \\
\hline \multicolumn{4}{|l|}{ Engrafted patients (\%) } \\
\hline Neutrophil & $29(100 \%)$ & $19(95 \%)$ & 0.40 \\
\hline Platelet & $25(86 \%)$ & $18(90 \%)$ & 0.69 \\
\hline \multicolumn{4}{|c|}{ Engraftment days (median, range) } \\
\hline Neutrophil & $15(10-30)$ & $15(12-22)$ & 0.80 \\
\hline Platelet & $13(9-66)$ & $22(9-79)$ & 0.36 \\
\hline \multicolumn{3}{|l|}{ CRS (Grade 1) } & \multirow[t]{3}{*}{0.04} \\
\hline No & $8(28 \%)$ & $1(5 \%)$ & \\
\hline Yes & $21(72 \%)$ & $19(95 \%)$ & \\
\hline \multicolumn{3}{|l|}{ Dexamethasone } & \multirow[t]{3}{*}{$<0.01$} \\
\hline No & $28(97 \%)$ & $5(25 \%)$ & \\
\hline Yes & $1(3 \%)$ & $15(75 \%)$ & \\
\hline \multicolumn{3}{|l|}{ Acute GVHD } & \multirow[t]{4}{*}{0.13} \\
\hline Grade 0-1 & $10(35 \%)$ & $13(65 \%)$ & \\
\hline Grade 2 & $10(35 \%)$ & $4(20 \%)$ & \\
\hline Grade 3-4 & $9(30 \%)$ & $3(15 \%)$ & \\
\hline \multicolumn{3}{|l|}{ Chronic GVHD } & \multirow[t]{5}{*}{0.59} \\
\hline No & $18(62 \%)$ & $15(70 \%)$ & \\
\hline Mild & $4(14 \%)$ & $3(18 \%)$ & \\
\hline Moderate & $4(14 \%)$ & $2(6 \%)$ & \\
\hline Severe & $3(10 \%)$ & $0(6 \%)$ & \\
\hline Complications & & & \\
\hline CMV viremia & $17(59 \%)$ & $6(30 \%)$ & 0.04 \\
\hline Hemorrhagic cystitis & $10(31 \%)$ & $4(20 \%)$ & 0.34 \\
\hline VOD & $4(14 \%)$ & $2(10 \%)$ & 0.69 \\
\hline Bleeding & $3(10 \%)$ & $1(5 \%)$ & 0.63 \\
\hline Adenovirus viremia & $2(7 \%)$ & $0(0 \%)$ & 0.50 \\
\hline Other & $5(17 \%)$ & $3(15 \%)$ & 0.83 \\
\hline Eltrombopag & $6(20 \%)$ & $4(20 \%)$ & 0.95 \\
\hline Relapse & $5(17 \%)$ & $7(35 \%)$ & 0.15 \\
\hline Death & $14(48 \%)$ & $7(35 \%)$ & 0.36 \\
\hline $\begin{array}{l}\text { Causes of Death } \\
\text { Infection }\end{array}$ & $4(14 \%)$ & $1(5 \%)$ & \\
\hline
\end{tabular}




$\begin{array}{lll}\text { Relapse/progression } & 2(7 \%) & 4(20 \%) \\ \text { aGVHD } & 2(7 \%) & 1(5 \%) \\ \text { Hemorrhage } & 2(7 \%) & 0(0 \%) \\ \text { Other/Unknown } & 4(14 \%) & 1(5 \%)\end{array}$

TBI: total body irradiation; GVHD: graft versus host disease; CNI: calcineurin inhibitor; MMF: mycophenolate mofetil; BM: bone marrow; PBSC: peripheral blood stem cell; TNC: total nucleated cell; MNC: mononucleated cell; CRS: Cytokine release syndrome; VOD: venoocclusive disease

Table 3: Results of Univariate Analysis for 2-year Outcomes

\begin{tabular}{|c|c|c|c|c|c|c|c|}
\hline & $\mathrm{n}$ & Pre-Cy (95 \% CI) & $\mathrm{p}$ & $\mathrm{n}$ & Post-Cy (95 \% CI) & $\mathrm{p}$ \\
\hline \multicolumn{2}{|l|}{ OS } & 29 & $55.2 \%(36.8-73.6)$ & & 20 & $56.8 \%(29.2-84.4)$ & 0.39 \\
\hline \multicolumn{2}{|l|}{ EFS } & 29 & $51.7 \%(33.1-70.3)$ & & 20 & $41.1 \%(14.1-68.1)$ & 0.97 \\
\hline \multicolumn{2}{|c|}{ CIR* } & 29 & $21.2 \%(9.7-46.5)$ & & 20 & $38.9 \%(22.6-66.8)$ & 0.33 \\
\hline \multicolumn{2}{|c|}{ OS (CR1 and CR2) } & 17 & $76.5 \%(55.9-97.1)$ & & 8 & $87.5 \%(64.1-100)$ & 0.53 \\
\hline \multicolumn{2}{|c|}{ EFS (CR1 and CR2) } & 17 & $70.6 \%(48.4-92.8)$ & & 8 & $75.0 \%(44.4-100)$ & 0.88 \\
\hline \multicolumn{2}{|c|}{ OS (CR3 and refractory) } & 12 & $25.0 \%(0-50.0)$ & & 12 & $41.7 \%(8.5-74.9)$ & 0.07 \\
\hline \multicolumn{2}{|c|}{ EFS (CR3 and refractory) } & 12 & $25.0 \%(0-50.0)$ & & 12 & $25.0 \%(0.0-53.8)$ & 0.30 \\
\hline \multirow[t]{2}{*}{ OS } & CRS (-) & 8 & $87.5 \%(64.1-100)$ & 0.04 & - & - & \\
\hline & CRS (+) & 21 & $42.9 \%(21.3-64.5)$ & & - & - & \\
\hline \multirow[t]{2}{*}{ EFS } & CRS (-) & 8 & $87.5 \%(64.1-100)$ & 0.04 & - & - & \\
\hline & CRS $(+)$ & 21 & $38.1 \%(16.9-59.3)$ & & - & - & \\
\hline \multirow[t]{2}{*}{ OS } & Dexamethasone (-) & - & - & & 5 & $100 \%(100-100)$ & 0.08 \\
\hline & Dexamethasone $(+)$ & - & - & & 15 & $42.9 \%(10.7-75.1)$ & \\
\hline \multirow[t]{2}{*}{ EFS } & Dexamethasone (-) & - & - & & 5 & $100 \%(100-100)$ & 0.03 \\
\hline & Dexamethasone $(+)$ & - & - & & 15 & $23.3 \%(0.0-49.9)$ & \\
\hline
\end{tabular}

* CIR: Cumulative incidence of relapse

\section{Figures}




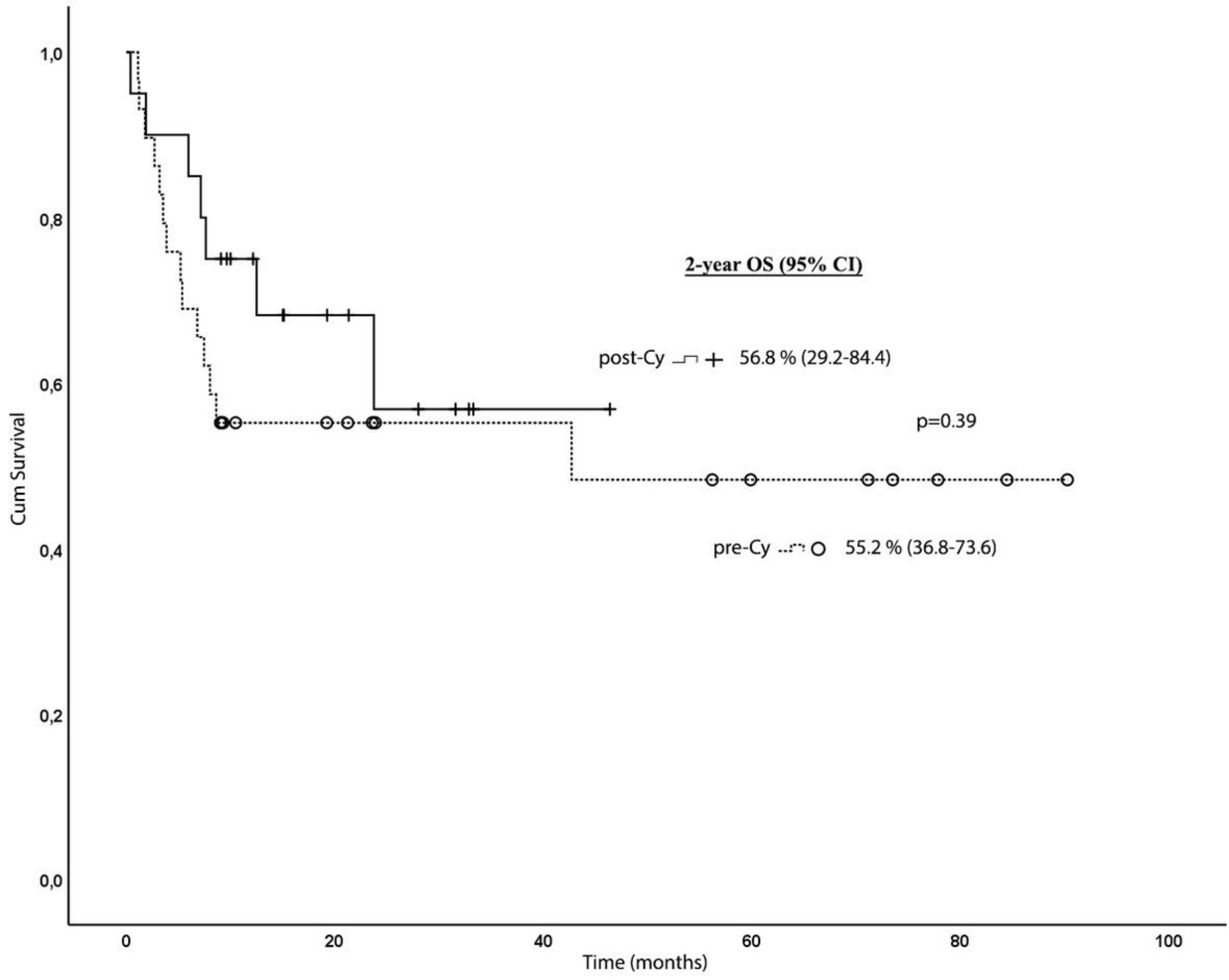

Figure 1

The 2 years probability of Overall survival 


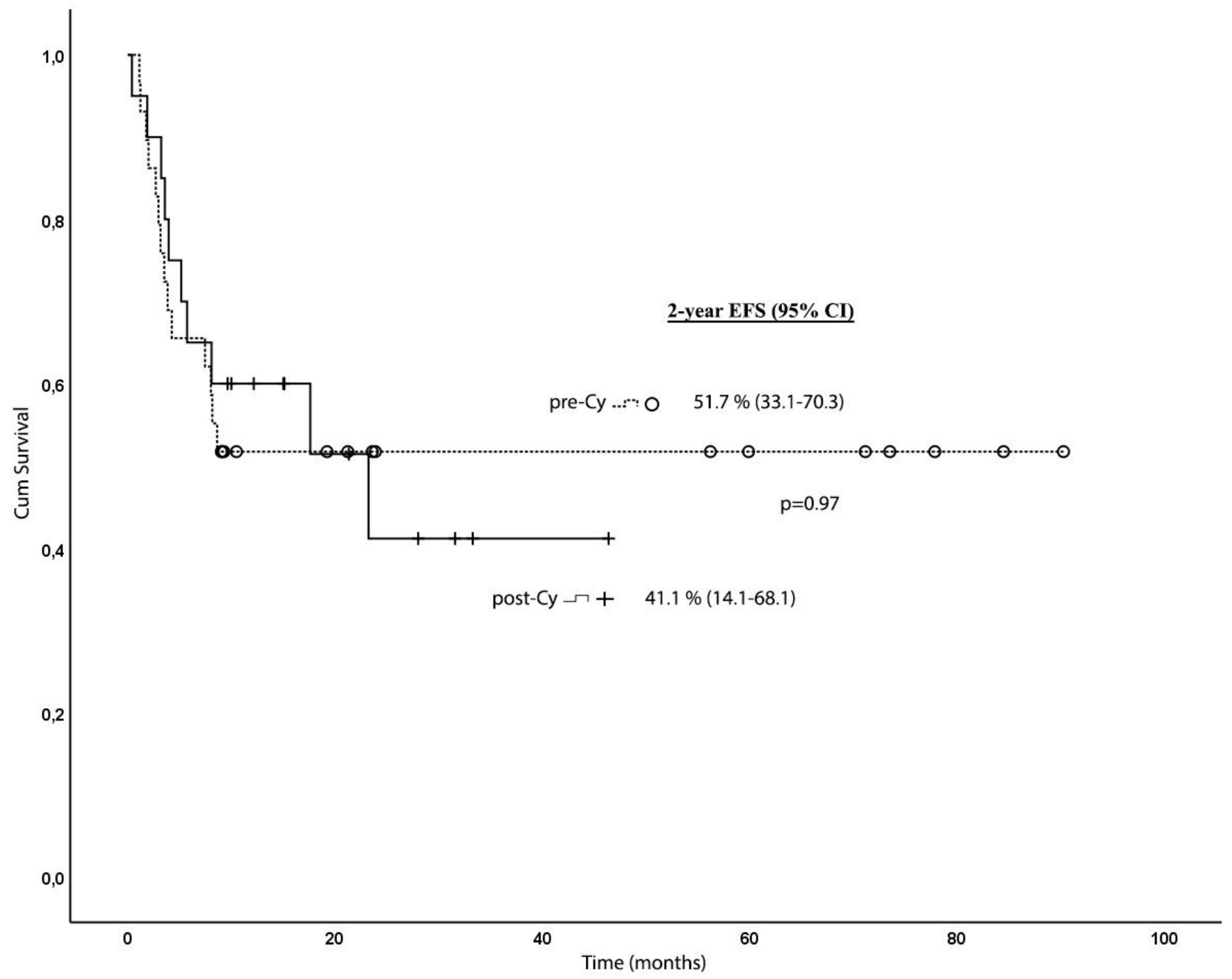

Figure 2

The 2 years probability of Event free survival 